\title{
A comparative study of intrathecal $0.5 \%$ isobaric levobupivacaine with fentanyl and $0.5 \%$ isobaric levobupivacaine with nalbuphine in infraumbilical surgeries: A randomized double blind clinical trial
}

\author{
Ramesh Koppal ${ }^{1}$, Dinesh Naik²*, Veeresh Mathpati ${ }^{3}$, Shivanand Hulkund ${ }^{4}$ \\ ${ }^{1}$ Professor, ${ }^{2}$ Assistant Professor, ${ }^{3}$ PG Student, ${ }^{4}$ Professor and HOD, Dept. of Anaesthesiology, S N Medical College, Bagalkot, Karnataka, \\ India \\ *Corresponding Author: Dinesh Naik \\ Email: dinesh.jnmc2002@gmail.com
}

Received: $14^{\text {th }}$ December, 2018

Accepted: $5^{\text {nd }}$ March, 2019

\begin{abstract}
Introduction: Spinal anaesthesia is most commonly used technique for lower abdominal surgeries. Its main disadvantage remains the short duration of action, hence different additives have been used. Opioids have been used to enhance the clinical efficacy and duration of action of local anesthetic drugs. The present study was aimed to compare the clinical efficiency of fentanyl and nalbuphine as an adjuvant to $0.5 \%$ Isobaric levobupivacaine for lower abdominal surgeries.

Materials and Methods: After obtaining ethical committee clearance and written consent from patient. ASA grade I\&II patients aged between 20-60 years undergoing infra umbilical surgeries were selected divided into two groups of 45 each by computerized randomization. Group A received intrathecal fentanyl $25 \mu \mathrm{g}(0.5 \mathrm{ml})$ with $3 \mathrm{ml}(15 \mathrm{mg}) 0 \mathrm{f} 0.5 \%$ Isobaric Levobupivacaine, and group B received intrathecal nalbuphine $0.8 \mathrm{mg}(0.5 \mathrm{ml})$ with $0.5 \%$ Isorbaric levobupivacaine $3 \mathrm{ml}(15 \mathrm{mg})$. Statistical analysis performed by STATA 11.2. Students $t$ test prior to find the significance difference between the study parameters.

Results: The duration of first rescue analgesia required prolonged in group B $(430.3 \pm 11.13 \mathrm{~min})$ compared to group A is $285.97 \pm 8.8 \mathrm{~min}$ with $\mathrm{p}$ value of $0.001(<0.05)$ (Table 1$)$. There were no significant hemodynamic changes and intra, postoperative complications in both groups.

Conclusion: Both the groups were equally efficacious with good intraoperative conditions with haemodynamic stability however group B improves the quality of intraoperative and postoperative analgesia with minimal side effects.
\end{abstract}

Keywords: Spinal anaesthesia, Nalbuphine, Levobupivacaine, Fentanyl, Postoperative analgesia.

\section{Introduction}

Spinal anaesthesia consists of the temporary interruption of nerve transmission within the subarachanoid space produced by injection of local anaesthetic solution into CSF. Used widely, safely and successfully spinal anaesthesia has many potential advantages over general anaesthesia, especially for operations involving the lower abdomen, the perineum and the lower extremities. ${ }^{1}$ Spinal anaesthesia is the most commonly used technique for infra umbilical surgeries because of its unmatchable reliability, cost effectiveness, effective analgesia, muscle relaxation and prolonged postoperative analgesia. Recent advances in anesthesia has allowed more surgeries to be performed on day case basis. ${ }^{2}$

The popularity of spinal block is that, the block has well defined end points and anaesthesiologist can produce the blocks relatively with a single injection. The versatility of spinal anaesthesia is afforded by a wide range of local anaesthetics and additives that allow control over the level, the time of onset and the duration of spinal anaesthesia. The distribution of local anaesthetic solutions within the subarachnoid space determines the extent of the neural blockade produced by spinal anaesthesia.

Levobupivacaine has been widely used in ambulatory surgeries after the development of low dose spinal anaesthesia technique. To improve the block characteristics of intrathecally administered low dose local anaesthetics, addition of adjuvant is must. ${ }^{3}$ Various types of medications can be used to overcome pain but opioids provide the most effective pain relief and standard of care. Intrathecal opioids are synergistic with local anaesthetics and intensify the sensory block without affecting the sympathetic block. ${ }^{4}$

Opioids are an important modality of postoperative pain management. They blunt the neuroendocrine stress response to pain. The technique of Opioid administration along with local anaesthetic has been exclusively studied to provide superior quality of analgesia in a variety of surgical procedures. 5 The rationale for the combination of opioids and local anaesthetics is that two types of drugs eliminate pain by acting at two different sites. Local anaesthetics act at the nerve axon and the opioid at the receptor site in the spinal cor. ${ }^{4,7}$

Thus this study is designed to quantitatively examine the effects of adding nalbuphine and fentanyl to $0.5 \%$ isobaric levobupivacaine hydrochloride spinal anaesthesia, to evaluate the efficacy, to know the duration of postoperative analgesia and to know the complications if any.

\section{Materials and Methods}

A prospective randomized, double blind clinical study was approved by our institutional ethical committee for a period of one year. Written informed consent was obtained from patients. 90 (45 in group A and other 45 in group B) patients of physical status American society of anesthesiologist (ASA) grade 1 and 2, aged between 20 to 60 
years undergoing elective infra umbilical surgeries under spinal anaesthesia were included in the study. Patients with history of known drug hypersensitivity to local anaesthetics and sensitivity to opioids, blood coagulation disorders, unwilling patients, spinal deformities, raised intracranial tension, local sepsis were excluded from the study.

A total sample size of 90 , sample size calculation done by using open epi software version 2.3.1. At 95\% confidence limit and $80 \%$ power of the study. According to study conducted by Nimisha P Brahmbhatt et al to evaluate the effects of fentanyl added to low dose levobupivacaine on sensory, motor blockade characteristics, postoperative analgesia \& complications, if any lower abdominal and perineal surgeries SD of motor block in Levobupivacaine plus Fentanyl group is found to be 16.05. Expecting / Assuming 10\% difference in motor block between two groups, sample size calculated is 41 45 in each group, hence the total sample size is 90 that is 45 in Group A and 45 in Group B.

\section{Materials and Methods}

A total of 90 patients undergoing elective infraumlical surgeries under subarachnoid block were included in the study. Patients randomly divided into two groups by using computer generated table.

Group A - will receive 15mg isobaric Levobupivicaine 0.5\% (3ml), plus $25 \mathrm{mcg}$ Fentanyl.

Group B - will receive 15mg isobaric Levobupivicaine $0.5 \%$ (3ml) plus $0.8 \mathrm{mg}$ Nalbuphine.

A detailed pre-anaesthetic evaluation will be carried out for each patient with relevant laboratory and radiological investigations. All patients will be visited a day prior to the surgery and explained in detail regarding the anaesthetic procedure and an informed written consent will be obtained.

All patients will receive tablet Ranitidine $150 \mathrm{mg}$ orally as pre- medication in the night. Patient will be kept nil per orally from 12 midnight prior to the day of surgery. On the day of surgery, patient will be preloaded with ringer lactate solution at the rate of $15 \mathrm{ml} / \mathrm{kg}$. Monitoring of vital parameters like heart rate, noninvasive arterial blood pressure, SPO2 and ECG will be done using automated multiparameter monitor. Basal line readings will be recorded.

Under aseptic precautions, spinal anaesthesia at L3-L4 interspace using $25 \mathrm{G}$ Quincke spinal needle with patient in sitting position would be performed. The studied drug would be injected into the subarachnoid space according to group, after noting the clear free flow of CSF with the operation table kept flat. Patients are turned supine immediately. Hypotension will be treated with intravenous incremental doses of Ephedrine $6 \mathrm{mg}$. Bradycardia will be treated with IV Atropine $0.6 \mathrm{mg}(0.02 \mathrm{mg} / \mathrm{kg}$ body weight $)$.

Sensory testing will be assessed by loss of pinprick sensation to $23 \mathrm{G}$ hypodermic needle for onset and dermatomal levels were tested every two minutes until, the highest level had stabilised for 4 consecutive tests. Testing will then be conducted every ten minutes until, the point of 2 segment regression of the block. Data regarding the time to reach highest dermatomal level of sensory blockade from the time of injection, time for 2 segment sensory regressions will be recorded. Sedation will be assessed intraoperatively by Ramsay sedation scale and recorded.

Group A - will receive $15 \mathrm{mg}$ isobaric Levobupivacaine $0.5 \%$ (3ml), 25mcg Fentanyl

Group B - will receive $15 \mathrm{mg}$ isobaric Levobupivacaine $0.5 \%$ (3ml) plus $0.8 \mathrm{mg}$ Nalbuphine.

After surgery, patient will be shifted to post anaesthesia care and recovery unit where they will remain until there is complete recovery of sensory and motor blockade. Postoperatively vital parameters will be recorded every hour and also any adverse events like nausea, vomiting, pruritus, shivering etc. will be noted. The time for rescue analgesia when patient complaints of pain at surgical site will be recorded and treated with suitable analgesics. Time to gain back the motor functions of lower limb defined as time to reach modified bromage 0 will also be noted.

\section{Parameters to be evaluated:}

1. Haemodynamic changes - HR, SBP, DBP, MAP, RR, SPO2, ECG will be monitored at every 5 minutes for 15 minutes and at every 10 min till the end of surgery. Then hourly in the postoperative period.

2. Assessment of motor blockade - Tested by modified Bromage scale.

Grade 0 : No motor block.

Grade 1: Inability to raise extended leg; able to move knees and feet.

Grade 2: Inability to raise extended leg and move knee; able to move feet.

Grade 3: Complete block of motor limb.

a. Duration of complete analgesia and effective analgesia would be assessed using visual analogue scale (VAS).

b. Side- effects and complications would be noted adverse effects like bradycardia, hypotension, respiratory depression, nausea, vomiting, shivering and pruritus will be noted.

\section{Statistical Analysis}

Data were entered in MS - Excel and analysed in SPSS V22. Descriptive statistics for qualitative data were represented with frequencies and percentages, whereas for quantitative data descriptive statistics were represented with Mean and Standard Deviation. Chi-square and t-test were applied for finding significance between the groups in qualitative and quantitative data respectively.

$\mathrm{P}<0.05$ was considered as statistically significant.

\section{Results}

Demographic data did not show any significant difference in age, weight, and sex ratio among the two groups and thus the two groups were comparable (Table 1). In our study the two groups were comparable with respect to their mean heart rate and the difference between the two groups were not statistically significant $(p>0.05)$ (Graph 1$)$ and also the groups were comparable with respect to their mean arterial pressure (MAP) because there was no 
statistically significance difference among the two groups with the $\mathrm{p}$ value of $0.641(\mathrm{p}>0.05)$ (Graph 2).

The mean duration of analgesia between the groups were statistically significant. There is significant difference among two groups in the maximum duration of analgesia with $p$ value of $0.001 \quad(p<0.05)$. (Table 2), and mean duration at which First Rescue Analgesic requirement between groups were statistically significant that is group A having at 277.6+_11.4 min compared to group B requiring at $431.0+\_13.5 \mathrm{~min}$ with $\mathrm{p}$ value of $0.001(<0.05)$. (Table 3 ). There is significant difference among two groups in maximum duration of motor block with $\mathrm{p}$ value of $0.001(\mathrm{p}<0.05)$. There was no statistically significant difference found between the groups for side effects. (Graph $3)$.

Table 1: Demographic data

\begin{tabular}{|c|c|c|}
\hline Variables & Group A (N=45) & Group B (N=45) \\
\hline Age (Years) & $52.96 \pm 13.06$ & $49.40 \pm 11.19$ \\
\hline Weight & $64.58 \pm 9.46$ & $67.76 \pm 7.60$ \\
\hline Gender (Male/Female) & $39 / 6$ & $41 / 4$ \\
\hline
\end{tabular}

Graph 1: Variation of mean heart rate between the groups

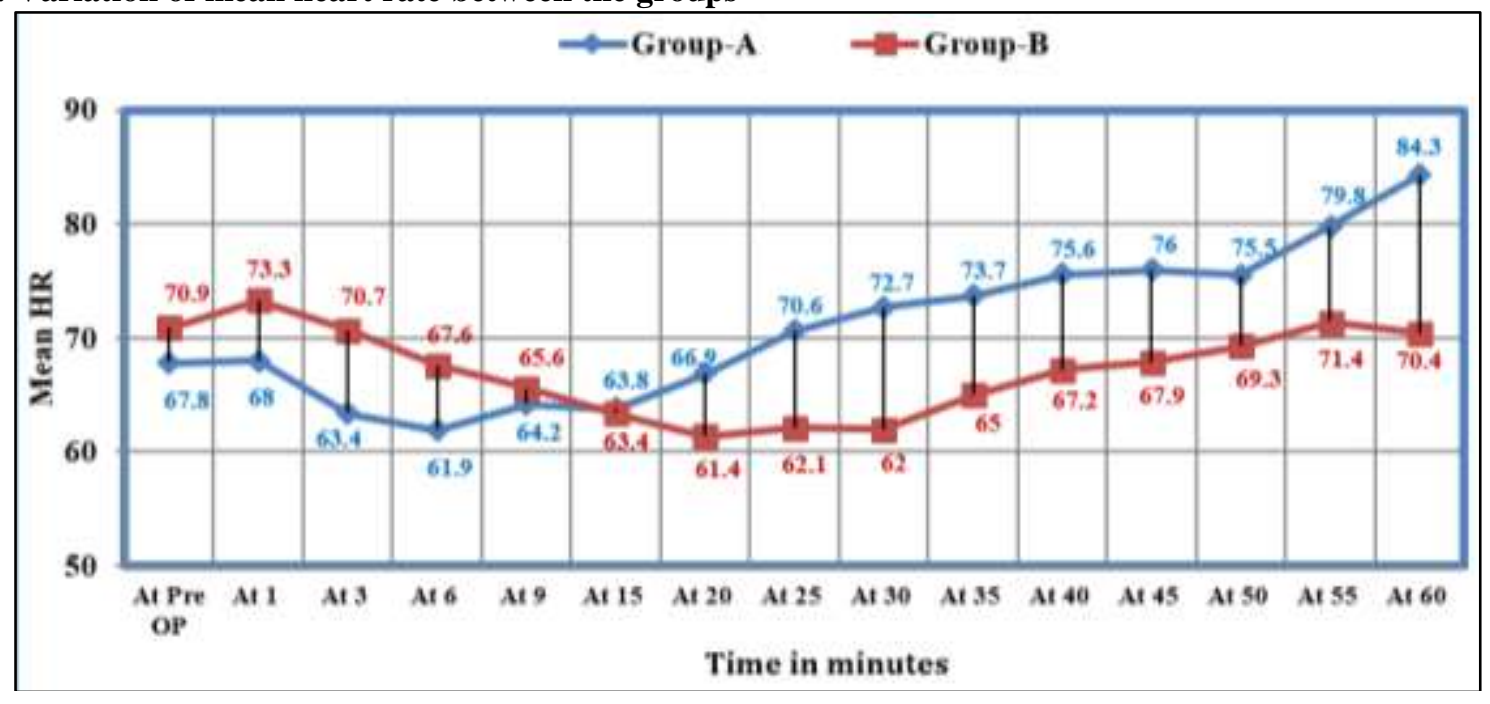

Graph 2: Graph showing variation of MAP between the groups

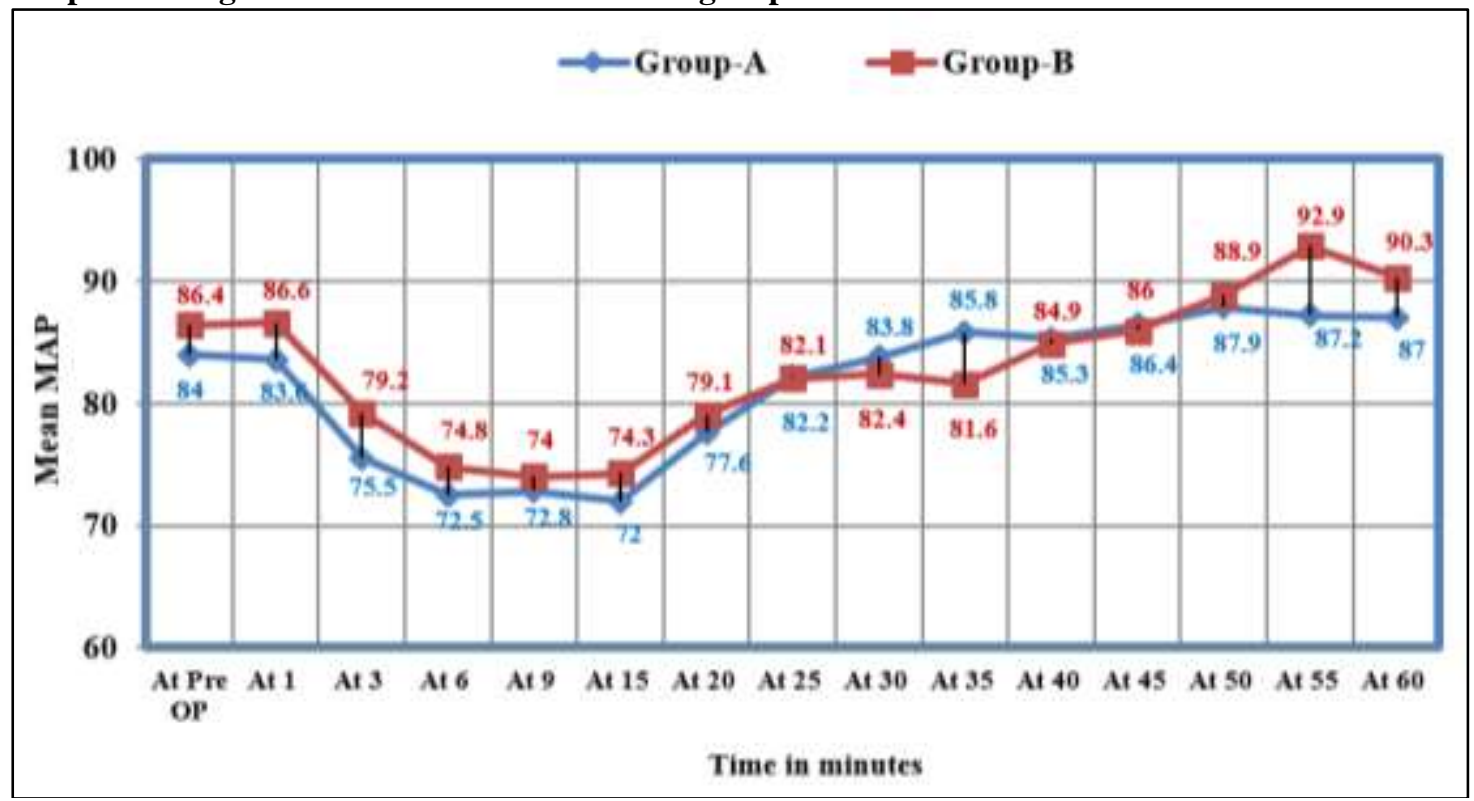


Table 2: Table showing duration of analgesia between groups:

\begin{tabular}{|l|c|c|c|c|}
\hline Variable & Group & Mean & SD & P-value \\
\hline $\begin{array}{l}\text { Duration of analgesia } \\
\text { (min). }\end{array}$ & $\mathrm{A}$ & 282.6 & 11.3 & $<0.001$ \\
\cline { 2 - 4 } & $\mathrm{B}$ & 436.9 & 13.0 & \\
\hline
\end{tabular}

Table 3: Table showing time of rescue analgesia between the groups

\begin{tabular}{|c|c|c|c|c|}
\hline \multicolumn{1}{|c|}{ Variable } & Group & Mean & SD & P-value \\
\hline $\begin{array}{l}\text { Rescue Analgesic } \\
\text { (min) post test }\end{array}$ & $\mathrm{A}$ & 277.6 & 11.4 & $<0.001$ \\
\cline { 2 - 4 } & $\mathrm{B}$ & 431.0 & 13.5 & \\
\hline
\end{tabular}

Table 4: Table showing duration of motor block between the groups:

\begin{tabular}{|l|c|c|c|c|}
\hline \multicolumn{1}{|c|}{ Variable } & Group & Mean & SD & P-value \\
\hline $\begin{array}{l}\text { Duration of Motor } \\
\text { Block(min) }\end{array}$ & A & 145.0 & 4.0 & $<0.001$ \\
\cline { 2 - 4 } & B & 138.8 & 9.9 & \\
\hline
\end{tabular}

Graph 3: Graph showing any complications between the groups

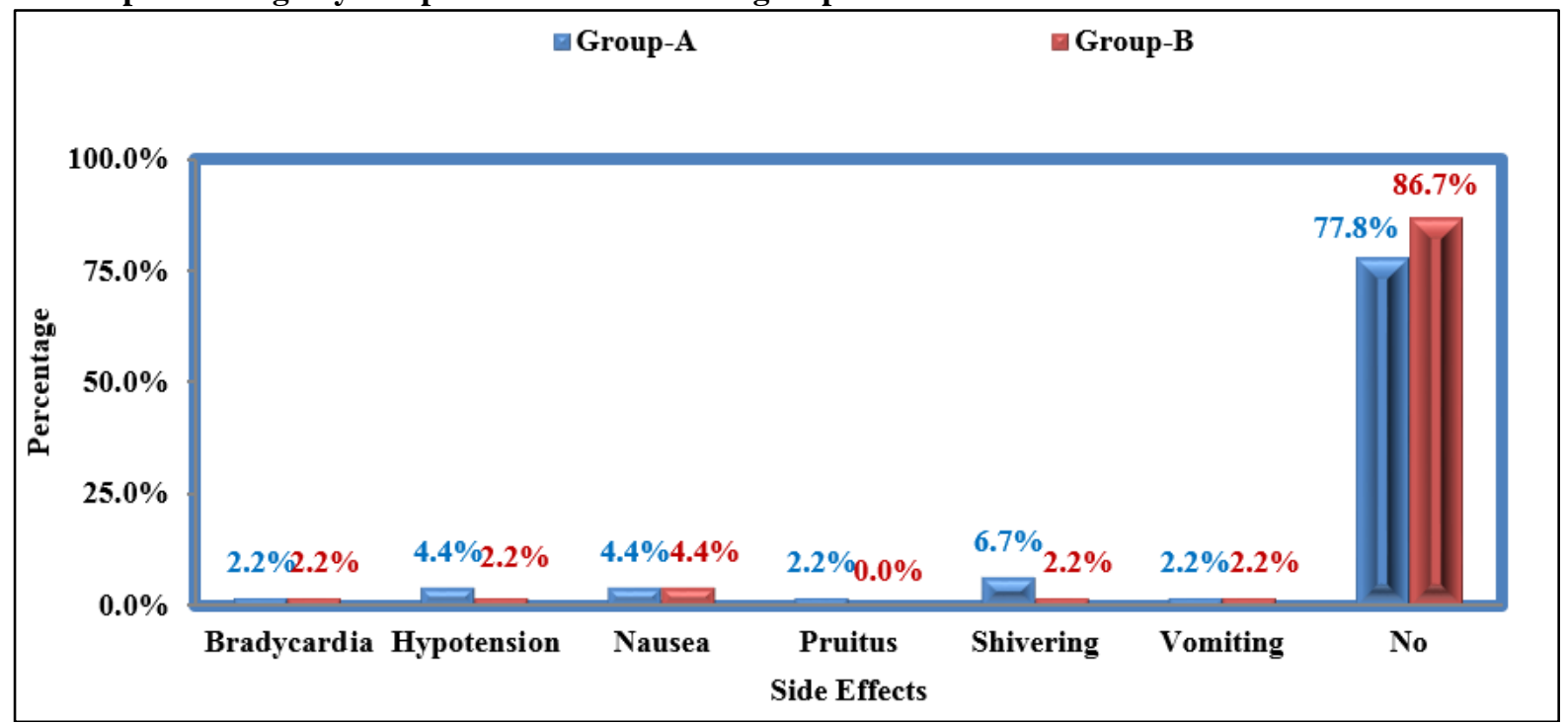

\section{Discussion}

Spinal anaesthesia is the most commonly used technique for infra umbilical surgeries because of its unmatchable reliability, cost effectiveness, effective analgesia, muscle relaxation and prolonged postoperative analgesia. Recent advances in anesthesia has allowed more surgeries to be performed on day case basis. Levobupivacaine has been widely used in ambulatory surgeries after the development of low dose spinal anaesthesia technique. To improve the block characteristics of intrathecally administered low dose local anaesthetics, addition of adjuvant is must. Intrathecal opioids enhance sensory block without prolonging motor and sympathetic block. Local anaesthetics work by inhibiting voltage-gated sodium channels in the spinal cord by interfering with afferent and efferent sensory and motor impulses while intrathecal opioids activate opioid receptors in the dorsal gray matter of the spinal cord (substantia gelatinosa) to modulate the function of afferent pain fibers. ${ }^{8}$ A number of adjuvants have been added to the intrathecal local anaesthetics for supplementation of intraoperative anaesthesia and postoperative analgesia. They have advantages as they reduce the dose of local anaesthetic; provide long lasting postoperative analgesia with reduced incidence of central nervous system depression, motor effects or hypotension. ${ }^{\text {? }}$

Opioid analogues have been used as additive to bupivacaine in spinal anaesthesia to improve the onset of action, quality of intraoperative and postoperative analgesia and to prolong the duration of block. Fentanyl is a potent synthetic opioid agonist and nalbuphine is a synthetic opioid agonist-antagonist analgesic. Some of intrathecal opioid absorbs back in the blood stream and produces analgesia by stimulating opioid receptors at brain level. Degree of this absorption is mainly determined by lipophilicity of the drug. Highly lipid soluble opioids like fentanyl or sufentanyl diffuse into blood stream quickly compared to less lipophilic morphine therefore producing short duration of analgesia. ${ }^{9}$ Highly hydrophilic opioids such as morphine, though provides very good intra and postoperative analgesia, its use becomes limited because of delayed 
respiratory depression that it causes due to rostral spread in intrathecal space. ${ }^{10}$

The present study was a randomized, prospective and double blind clinical study, done in 90 patients belonging to the age group of 20-60 year female patients of ASA grade I and II undergoing for infra umbilical surgeries. It was designed to compare the effects of adding inrathecal nalbuphine and fentanyl as an adjunct to levobupivacaine in spinal anaesthesia by assessing the motor blockade characteristics and duration of postoperative analgesia. There are limited data on comparison of spinal effects of nalbuphine and fentanyl. ${ }^{9}$

Fentanyl acts by binding with opioid receptors in the dorsal horn of spinal cord and may also have its action via supra spinal spread when given intrathecally and has been used as an adjuvant to local anaesthetics in subarachnoid block. It reduces both visceral and somatic pain but its use is now limited due to dose dependent adverse effects associated with it. ${ }^{11,12}$

Singh $\mathrm{H}$, et al $1^{13}$ in 1995 , Biswas BN, et al ${ }^{14}$ in 2002 , Khanna MS, et $\mathrm{al}^{15}$ in 2002 have chosen 25 micrograms of fentanyl as an additive to intrathecal hyperbaric bupivacaine in their studies. Hence in our study, we chose 25 micrograms of fentanyl as an additive to levobupivacaine.

Nalbuphine is a synthetic lipophilic opioid with agonist action at the kappa opioid receptor and antagonist at the mu receptor. Unlike morphine, it has a short duration of action due to its liposolubility and rapid plasma clearance ${ }^{9}$. Mechanism of analgesia is by its agonistic action on this receptor. It also stimulates kappa receptors. This inhibits release of neurotransmitter that mediates pain such as substance P. In addition it acts as post synaptic inhibitor on the interneuron and output neuron of spinothalamic tract which transports nociceptive information. It improves quality of block and offers prolonged and long lasting postoperative analgesia. It has low incidence of adverse effects known for other opioids (respiratory depression, nausea, vomiting, pruritus). It is also cost effective. ${ }^{16}$ There are only few studies available of central neuraxial administration of nalbuphine as intrathecal adjuvant, which concluded that nalbuphine significantly enhanced the sensory analgesia with minimal pruritus and respiratory depression. ${ }^{17}$

In our study mean duration of first rescue analgesic requirement in group A is at $285.97+8.8 \min \&$ in group B is at $430.3+11.13 \mathrm{~min}$ with $\mathrm{p}$ value of $0.001(<0.05)$ which is statistically significant. And this is similar to study done by Gupta $\mathrm{K}$, et $\mathrm{al}^{8}$ in which they compared intrathecal $0.5 \%$ hyperbaric bupivacaine $17.5 \mathrm{mg}(3.5 \mathrm{ml})$ with fentanyl 25 mcg and $0.5 \%$ hyperbaric bupivacaine $17.5 \mathrm{mg}(3.5 \mathrm{ml})$ with nalbuphine $2 \mathrm{mg}$ in lower limb orthopaedic surgeries showing mean time for first analgesic requirement is at $278.74+29.67 \mathrm{~min}$ in FB group and in group NB is at $318.64+21.92 \mathrm{~min}$ with $\mathrm{p}$ value of $0.000(<0.05)$ which is statistically significant. And also similar to study done by Bisth, et $\mathrm{al}^{9}$ in which they compared the intrathecal $0.5 \%$ hyperbaric bupivacaine $15 \mathrm{mg}$ with fentanyl $25 \mathrm{mcg}(0.5 \mathrm{ml})$ and $0.5 \%$ hyperbaric bupivacaine $15 \mathrm{mg}$ with nalbuphine
$1 \mathrm{mg}$ in total abdominal hysterectomy surgeries in which they concluded that mean time for first rescue analgesic requirement in FB group is at 283.44+_78.97 min and in NB group is at $460.78+77.98$ min with $p$ value of $<0.001(<0.05)$ which is statistically significant

In our study mean duration of motor block in group A $145.0+\_4 m i n$ \&in group B 138.8+_9.9 with p value of $0.001(<0.05)$ statistically significant which is similar to the study done by Gupta $\mathrm{K}$, et $\mathrm{al}^{8}$ in which they compared the intrathecal bupivacaine with fentanyl $(25 \mathrm{mcg})$, nalbuphine $2 \mathrm{mg}$, showing mean duration of motor blockade in FB group141.63+_18.05min and in BN group 183.26+_21.92 min ( $\mathrm{p}$ value 0.003 ), stastically significant.

The present study revealed no statistically significant difference in the cardiovascular parameters like mean heart rate, mean systolic blood pressure, mean diastolic blood pressure, mean arterial pressure, $\mathrm{sp} 02$ and haemodynamic status and our study was comparable to Gupta $\mathrm{K}$ et $\mathrm{al}^{8}$ and Culebras $\mathrm{X}$ et $\mathrm{al}^{18}$ for haemodynamic parameters.

In the present study, two patients developed hypotension in group $\mathrm{A}$ and one patient in group B, this could be due to $0.5 \%$ isobaric levobupivacaine $(15 \mathrm{mg}$ ) in subarachnoid space producing hypotension rather than opioids. Nausea developed in 2 patients in each group, shivering developed in 3 patients in group A and one patient in group B, there was no significant statistical difference in the adverse events between the two groups.

Similar finding was seen in study done by Culebras X, et $\mathrm{al}^{18}$ comparing the $0.5 \%$ hyperbaric bupivacaine $15 \mathrm{mg}$ with fentanyl $25 \mathrm{mcg}$ and $0.5 \%$ hyperbaric bupivacaine 15 $\mathrm{mg}$ with nalbuphine $1 \mathrm{mg}$ in lower abdominal surgeries In fenatnyl Group two patients developed hypotension and one had pruritus. Nausea was seen in two patients in either group. None developed respiratory distress. As far as side effects of intrathecal opioids were concerned in our study, patients in both groups had minimal side effects. No pruritus, respiratory depression, euphoria dysphoria, desaturation in both the groups.

\section{Conclusion}

Both the groups were equally efficacious with good intra operative conditions with haemodynamic stability however group B improves the quality of intra operative and postoperative analgesia with minimal side effects.

\section{Conflict of Interest: None.}

\section{References}

1. Christopher M. Bernards: Epidural and spinal anaesthesia in: Paul G. Barash Clinical anaesthesia $6^{\text {th }}$ edition Wolster Kluwer Lippioncot Williams \& Wilkins New York 2010:927-950.

2. Attri JP, Kaur J, Kaur S, Kaur R, Mohan B, Kashyap K. Comparison of levobupivacaine and levobupivacaine with fentanyl in infra umbilical surgeries under spinal anaesthesia. Anesth Essays Res 2015;9:178-84.

3. Brahmbhatt NP, Prajapati IA, Upadhyay MR. Combination of Low Dose Isobaric Levobupivacaine $0.5 \%$ and Fentanyl Compared with Isobaric Levobupivacaine $0.5 \%$ in Spinal Anaesthesia for Lower Abdominal and Perineal Surgeries. Int J Res Med 2015; 4(2);55-60. 
4. Gomaa HM, Mohamed NN. A comparison between postoperative analgesia after intrathecal nalbuphine with bupivacaine and intrathecal fentanyl with bupivacaine after cesarean section. In: Egypt J Anaesth Elsevier. 2014:05:410.

5. Mark A, Chaney MD. Side effects of intrathecal and epidural opioids. Can J Anesth 1995(42);10:891-903.

6. Jyothi B, Shaikh S and Gowda S. A comparison of analgesic effect of different doses of intrathecal nalbuphine hydrochloride with bupivacaine and bupivacaine alone for lower abdominal and orthopedic surgeries. Indian J Pain 2014;28:18-23.

7. Schneider M, Ettlin T, Kaufmann M. Transient neurologic toxicity after hyperbaric subarachnoid anesthesia with 5\% lidocaine. Anesth Analg 1993;76:1154-7.

8. Gupta K, Rastogi B, Gupta PK, Singh I, Bansal M. and Tyagi $\mathrm{V}$. Intrathecal nalbuphine versus intrathecal fentanyl as adjuvant to $0.5 \%$ hyperbaric bupivacaine for orthopedic surgery of lower limbs under subarachnoid block: A comparative evaluation. Indian J Pain 2016;30:90-5.

9. Bisht S, Rashmi D. Comparison of intrathecal fentanyl and nalbuphine: A prospective randomized controlled study in patients undergoing total abdominal hysterectomy. Anaesth Pain Intensive Care 2017;21(2):194-8.

10. Biswas BN, Rudra A, Bose BK, Nath S, Chakrabarty S, Bhattacharjee S. Intrathecal fentanyl with hyperbaric bupivacaine improves analgesia during caesarean delivery and in early postoperative period. Indian J. Anaesth 2002;46(6):469-72.

11. Al-Mustafa MM, Abu-Halaweh SA, Aloweidi AS, Murshidi MM, Ammari BA, Awwad ZM, et al. Effect of dexmedetomidine added to spinal bupivacaine for urological procedures. Saudi Med J 2009;30(3):365-70.

12. Benhamou D, Thorin D, Brichant JF, Dailland P, Milon D, Schneider M. Intrathecal clonidine and fentanyl with hyperbaric bupivacaine improves analgesia during cesarean section. Anesth Analg 1998;87(3):609-13.
13. Singh H, Yang J, Thornton K, Giesecke AH. Intrathecal fentanyl prolongs sensory bupivacaine spinal block. Can J Anaesth 1995;42(11):987-91.

14. Biswas BN, Rudra A, Saha JK, Karmakar S. Comparative study between effects of intrathecal midazolam and fentanyl on early postoperative pain relief after inguinal herniorrhaphy. $J$ Anaesth Clin Pharmacol 2002;18(3):280-3.

15. Khanna MS, Singh IKJP. Comparative evaluation of bupivacaine plain versus bupivacaine with fentanyl in spinal anaesthesia in geriatric patients. Indian J Anaesth 2002;46(3):199-203.

16. Sapate M, Sahu P, Thatte WS, Dubey R. A randomized, double blind, control study of the effects of adding nalbuphine to spinal bupivacaine for lower abdominal surgeries in elderly patients. Anaesth Pain Intensive Care 2013;17(2):145-8.

17. Naaz S, Shukla U, Srivastava S, Ozair E, Asghar A. A Comparative Study of Analgesic Effect of Intrathecal Nalbuphine and Fentanyl as Adjuvant in Lower Limb Orthopaedic Surgery. J Clin Diagn Res 2017;11(7): UC25UC8.

18. Culebras X, Gaggero G, Zatloucal J, Kern C, Marti RA. Advantages of Inrathecal Nalbuphine, compared with Intrathecal Morphine, after caesarean delivery: An evaluation of postoperative Analgesia and Adverse effects. Anesth Analg 2000;91:601-5.

How to cite this article: Koppal R, Naik D, Mathpati V, Hulkund S. A comparative study of intrathecal $0.5 \%$ isobaric levobupivacaine with fentanyl and $0.5 \%$ isobaric levobupivacaine with nalbuphine in infraumbilical surgeries: A randomized double blind clinical trial. Indian J Clin Anaesth 2019;6(2):203-8. 